\title{
Untersuchungen zur Wachstumsprognose asthmakranker Kinder
}

\author{
W. F. Baum ${ }^{1}$ \\ F. B. Kosubek ${ }^{1}$ \\ U. Schneyer ${ }^{2}$ \\ E. Klöditz ${ }^{1}$
}

\author{
Investigation of Growth Prognosis in Asthmatic Children
}

\section{Zusammenfassung}

Ziel der vorliegenden Arbeit war die Ermittlung aktueller Daten zur Wachstumsprognose asthmakranker Kinder. Dazu wurden bei 210 Asthmatikern - 153 Jungen und 57 Mädchen - im Alter von 1,7 bis 18,9 Jahren die Parameter Lebens(CA)-, Knochen(KA)und Längenalter (LA), Körperhöhe (KH), Erkrankungsschweregrad, genetische Zielgröße (GZG) und prognostische Endgröße (PEG) bestimmt.

5,9\% der Jungen und 3,5\% der Mädchen erwiesen sich als kleinwüchsig $(\mathrm{KH}<\overline{\mathrm{X}}-2 \mathrm{~s})$. Das entspricht etwa dem 2,5- bzw. 1,5fachen einer Normalverteilung. Kinder mit dem höchsten Erkrankungsschweregrad zeigten die stärkste negative Abweichung der KH-SDS-Werte (Jungen $-0,59 \pm 1,1$, Mädchen $-0,97 \pm 0,8$ ), die beim weiblichen Geschlecht signifikant ausfiel. Die Entwicklung von CA, KA und LA verlief bis zum 4. Lebensjahr unauffällig. $\mathrm{Ab}$ dem 5. Lebensjahr konnte bei beiden Geschlechtern eine zum Teil signifikante Retardierung des KA und LA festgestellt werden. Bis zum 16. Lebensjahr war das KA, danach das LA stärker betroffen. Ein Vergleich der GZG- und PEG-Mittelwerte innerhalb der Schweregradgruppen machte deutlich, dass die PEG bei beiden Geschlechtern, unabhängig vom Erkrankungsschweregrad, ausnahmslos kleiner war als die GZG. Die Differenzen beider Größen erwiesen sich für die Schweregradgruppe 3 der Jungen (180,8 \pm $6,5 \mathrm{~cm}$ zu $175,8 \pm 6,2 \mathrm{~cm}, \mathrm{p}<0,01$ ) und für die Gesamtgruppen beider Geschlechter (Jungen $180,2 \pm 5,3 \mathrm{~cm}$ zu $178,6 \pm 7,3 \mathrm{~cm}$, $\mathrm{p}<0,05$, Mädchen $167,7 \pm 4,4 \mathrm{~cm}$ zu $165,8 \pm 6,8 \mathrm{~cm}, \mathrm{p}<0,05)$ als signifikant. Bei einem getrennten Vergleich der GZG- bzw. PEGMittelwerte zwischen den Schweregradgruppen zeigte sich dagegen keine signifikante Differenz.

Die Ergebnisse sprechen für einen wachstumshemmenden Einfluss des Asthma bronchiale. Als Ursache müssen der Erkrankungsschweregrad, aber auch direkte Auswirkungen der Atopie

\section{Abstract}

The objective of the present study was the ascertainment of correct data related to growth prognosis in asthmatic children. For this purpose, 210 young asthmatics including 153 males and 57 females ranging in age from 1.7 to 18.9 years were evaluated. Data were specified for the parameters: chronological age (CA); bone age (KA); height age (LA); height (KH); severity of illness; target adult height (GZG) and predicted adult height (PEG).

Among this group, $5.9 \%$ of the boys and $3.5 \%$ of the girls, were found to be of small stature $(\mathrm{KH}<\overline{\mathrm{x}}-2 \mathrm{~s})$. This corresponds to about 2.5 and 1.5 times the average in a normal distribution. Children with the highest severity of illness showed the strongest negative deviation for the KH-SDS values (boys $-0.59 \pm 1.1$, girls $-0.97 \pm 0.8$. This proved to be statistically significant for females. Development of CA, KA and LA continued unremarkably until the age of 4 years. From age 5, partially a significant growth retardation of KA and LA could be observed in both sexes. To age 16, KA values and, after that LA values, were more strongly affected. Comparison of the GZG and PEG average values within the degree of severity groups made it clear that the PEG values for both sexes, independent of the severity of illness, were without exception, smaller than the GZG. The differences of both parameters proved to be statistically significant for the group with the most severe symptoms of the boys $(180.8 \pm 6.5 \mathrm{~cm}$ to $175.8 \pm 6.2 \mathrm{~cm}, \mathrm{p}<0.01$ ) and for the entire groups of both sexes (boys $180.2 \pm 5.3 \mathrm{~cm}$ to $178.6 \pm 7.3 \mathrm{~cm}, \mathrm{p}<0.05$, girls $167.7 \pm$ $4.4 \mathrm{~cm}$ to $165.8 \pm 6.8 \mathrm{~cm}, \mathrm{p}<0.05$ ). However, a separate comparison of GZG and PEG average values, respectively, among the severity groups showed no significant differences.

The results argue for a growth-inhibiting influence from bronchial asthma. The cause has to be severity of illness but direct effects of the atopy on skeletal development is also taken in con- 
auf die Skelettentwicklung in Betracht gezogen werden. Zur Bestätigung dieser Befunde wären die Untersuchung einer größeren Probandengruppe und die Abklärung pathophysiologischer Abläufe am wachsenden Skelett atopischer Kinder erforderlich. sideration. Confirmation of these findings requires investigation of a larger group of asthmatics and clarification of the pathophysiological processes in the growing skeletons of atopic children.

\section{Einleitung}

Das gehäufte Auftreten von Wachstums- und Entwicklungsstörungen asthmakranker Kinder wird im Allgemeinen auf einen schweren Krankheitsverlauf [1 -5] bzw. eine inadäquate Glukokortikoid-Therapie [6-11] zurückgeführt. Zweifelsohne spielen diese Faktoren eine wichtige Rolle. Sie sind aber keineswegs als alleinige Ursache anzusehen. Dafür sprechen Beobachtungen, nach denen auch Patienten mit leichten Asthmaverläufen, die bislang noch nicht mit Glukokortikoiden behandelt worden sind, erhöhte Kleinwuchsraten aufweisen [2,4,6,12 - 14]. Diese Befunde sind als Hinweis auf eine möglicherweise kausale Bedeutung der dem Asthma bronchiale oft zugrunde liegenden allergischen Disposition, der Atopie, interpretiert worden [3,5,15-17].

Bestimmungen des Knochenalters ergaben teilweise deutliche Verzögerungen, so dass die asthmaassoziierte Kleinwüchsigkeit auf einer Skelettretardierung zu basieren scheint $[1,4,15,17-19]$. Es ist unklar, ob es sich bei diesem Befund lediglich um ein Symptom oder aber die eigentliche Ursache der Entwicklungsverzögerung handelt. Eigene Beobachtungen sprechen für das Vorliegen einer primären atopischen Skelettmanifestation und damit eine vom Asthma bronchiale eher unabhängige Beeinflussung des Knochenwachstums [17]. Durch einen schweren Asthmaverlauf können Wachstum und Entwicklung zusätzlich behindert werden.

Als markantes klinisches Symptom der Entwicklungsverzögerung ist ein verspäteter Pubertätsbeginn beschrieben worden $[5,18,20,21]$. Die Wachstumsprognose wird jedoch als gut bezeichnet. Betroffene Kinder sollen eine normale Erwachsenengröße erreichen $[8,18,20,22,23]$. Für Asthmatiker mit leichtem Krankheitsverlauf ist sogar eine über dem Altersdurchschnitt liegende Erwachsenenendgröße ermittelt worden [24].

Eine objektive Bewertung der prognostizierten bzw. nach Abschluss des Wachstums direkt ermittelten Endgröße ist allerdings nur unter Berücksichtigung der genetischen Zielgröße möglich. Untersuchungen dieser Art liegen vereinzelt vor. Sie bestätigen die gute Wachstumsprognose [25 - 27]. Bei der Berechnung der genetischen Zielgröße ist die Akzeleration allerdings nicht immer ausreichend berücksichtigt worden. Das kann zu Fehlinterpretationen des Asthmaeinflusses auf die Endgröße führen.

Ziel der vorliegenden Arbeit war die Ermittlung akuteller Daten zur Wachstumsprognose asthmakranker Kinder. Insbesondere sollte durch Vergleich von genetischer Ziel- und prognostischer Endgröße der Einfluss des Asthma bronchiale bzw. des Erkrankungsschweregrades und der Atopie auf Wachstum und Entwicklung überprüft werden.

\section{Patienten und Methodik}

In die Studie wurden 210 asthmakranke Kinder - 153 Jungen und 57 Mädchen - im Alter von 1,7 bis 18,9 Jahren einbezogen. Das Vorliegen atopischer Begleiterkrankungen, wie Neurodermitis oder Rhinitis allergica, wurde toleriert. Chronische Erkrankungen anderer Genese, Endokrinopathien oder Kleinwuchs der Eltern führte dagegen zum Ausschluss aus dem Untersuchungsprogramm. Von jedem Patienten wurden Lebens-, Knochenund Längenalter, Körperhöhe, Erkrankungsschweregrad und die mittlere Elterngröße erfasst.

Das chronologische Alter (CA) wurde als Dezimalzahl angegeben. Zur statistischen Auswertung wurden Vierjahresaltersgruppen gebildet: AG 1: 1-4 Jahre, AG 2: 5-8 Jahre, AG 3: 9-12 Jahre, AG 4: 13 - 16 Jahre, AG 5: $\geq 17$ Jahre).

Die Körperhöhe $(\mathrm{KH})$ wurde nach den Kriterien von Martin und Saller [28] mit einem Anthropometer bestimmt. Kinder unter 2 Jahren wurden im Liegen in einer Messmulde gemessen. Die Messgenauigkeit lag bei $0,1 \mathrm{~cm}$. Für die $\mathrm{KH}$ wurden SDS-Werte (standard deviation score) berechnet. Sie ergeben sich aus der Formel SDS $=\mathrm{x}-\overline{\mathrm{x}}: \mathrm{s}$, wobei $\mathrm{x}$ der gemessene Individualwert, $\overline{\mathrm{x}}$ der Mittelwert einer normalen Vergleichsgruppe und s die dazugehörige Standardabweichung darstellt. SDS-Werte erlauben unter der Voraussetzung der Normalverteilung eine altersunabhängige Bewertung der gemessenen Variablen.

Die Bestimmung des Knochenalters (KA) erfolgt anhand einer Röntgenaufnahme der linken Hand nach der von Tanner u. Mitarb. angegebenen 20-Bone-Methode [29]. Für Knaben im Alter von 4,0 bis 16,5 Jahren und Mädchen zwischen 4,5 und 15,5 Jahren wurden von Tanner u. Mitarb. Koeffizienten mitgeteilt, die nach der Formel Erwachsenenendgröße $=(\mathrm{k} 1 \times \mathrm{KH})-$ $(\mathrm{k} 2 \times \mathrm{CA})-(\mathrm{k} 3 \times \mathrm{KA})+\mathrm{K}$ die Berechnung der prognostischen Endgröße (PEG) ermöglichen. Dazu ist die KA-Bestimmung nach der ebenfalls von Tanner u. Mitarb. inaugurierten RUSMethode (Radius, Ulna, short bones) erforderlich, so dass das KA für diese Altersgruppen zusätzlich auch auf diese Weise bestimmt worden ist.

Unter Längenalter (LA) ist das Alter zu verstehen, bei dem die akutelle KH der 50. Perzentile - und damit der Durchschnittsgröße der Normalpopulation - entspricht. KA und LA ermöglichen als gut vergleichbare Entwicklungsparameter eine recht verlässliche Abschätzung des körperlichen Entwicklungsstandes.

Abweichend vom derzeit international üblichen Einteilungsschema in 4 Schweregrade, das vor allem Bedeutung für die Therapiegestaltung besitzt, wurde für die vorliegende Arbeit eine gröbere Klassifikation in die Schweregrade 1 (leicht, keine systemischen Glukokortikoide), 2 (mittelschwer, systemische Glukokortikoide bis zu 3 Mon./Jahr) und 3 (schwer, systemische Gluko- 
kortikoide länger als $3 \mathrm{Mon}$./Jahr) vorgenommen, da diese für die zu bearbeitende Problematik als aussagekräftiger anzusehen ist. Als Bewertungsgrundlage diente der medikamentöse Therapiebedarf der letzten ein bis zwei Jahre.

Die KH der Eltern wurde während der Asthma-Sprechstunde gemessen. Eltern, die auf diese Weise nicht erfasst werden konnten, wurden angeschrieben und gebeten, ihre $\mathrm{KH}$ möglichst in einer Messgenauigkeit von $0,1 \mathrm{~cm}$ mitzuteilen. Das arithmetische Mittel der Größe beider Eltern, die sogenannte mittlere Elterngröße, diente als Basis für die Bestimmung der genetischen Zielgröße (GZG). Diese errechnet sich aus der mittleren Elterngröße zuzüglich 10,2 cm für Knaben und abzüglich 2,6 cm für Mädchen [30]. Da die Koeffizienten zur Bestimmung der PEG nur für bestimmte Altersgruppen vorliegen (siehe oben) und die mittlere Elterngröße nicht in allen Fällen zu ermitteln war, konnten vergleichende Untersuchungen zur Wachstumsprognose nur bei 99 Knaben und 30 Mädchen vorgenommen werden.

Die statistische Auswertung erfolgte mit Hilfe der einfaktoriellen Varianzanalyse, des Friedman- und des Wilcoxon-Tests. Die Irrtumswahrscheinlichkeit wurde bei $5 \%$ angenommen. Als Referenzdaten zur Berechnung der KH-SDS-Werte dienten die von Hesse mitgeteilten Normwerte [31]. Planung und Durchführung der Untersuchungen erfolgten nach den Prinzipien der HelsinkiDeklaration 1989. Die Röntgenaufnahmen der Hand wurden in den Jahren 1989 und 1990 angefertigt. Zu diesem Zeitpunkt war die Berufung einer Ethikkommission an der Medizinischen Fakultät der Martin-Luther-Universität Halle-Wittenberg noch nicht erfolgt.

\section{Ergebnisse}

Die Mehrzahl asthmakranker Kinder war körperlich normal entwickelt. Jedoch wiesen 9 Jungen (5,9\%) und 2 Mädchen (3,5\%) eine Körperhöhe $<\bar{x}-2 x$ auf und waren somit als kleinwüchsig einzustufen (siehe Abb.1 und 2). Die Kleinwuchsrate liegt damit etwa um das 2,5- bzw. 1,5fache höher als in einer Normalpopulation. Das Ausmaß der Wachstumsretardierung scheint in gewissem Umfang vom Erkrankungsschweregrad abhängig zu sein. Asthmatiker der Schweregradgruppen 3 zeigten mit mittleren KH-SDS-Werten von $-0,59 \pm 1,12$ (Knaben) bzw. $-0,97 \pm 0,81$ (Mädchen) jeweils die größte negative Abweichung (siehe Tab.1). Die Unterschiede zwischen den Schweregraden waren beim weiblichen Geschlecht für die KH-SDS-Werte, aber - als Hinweis auf eine inhomogene Altersverteilung in den Gruppen - auch für die KH signifikant.

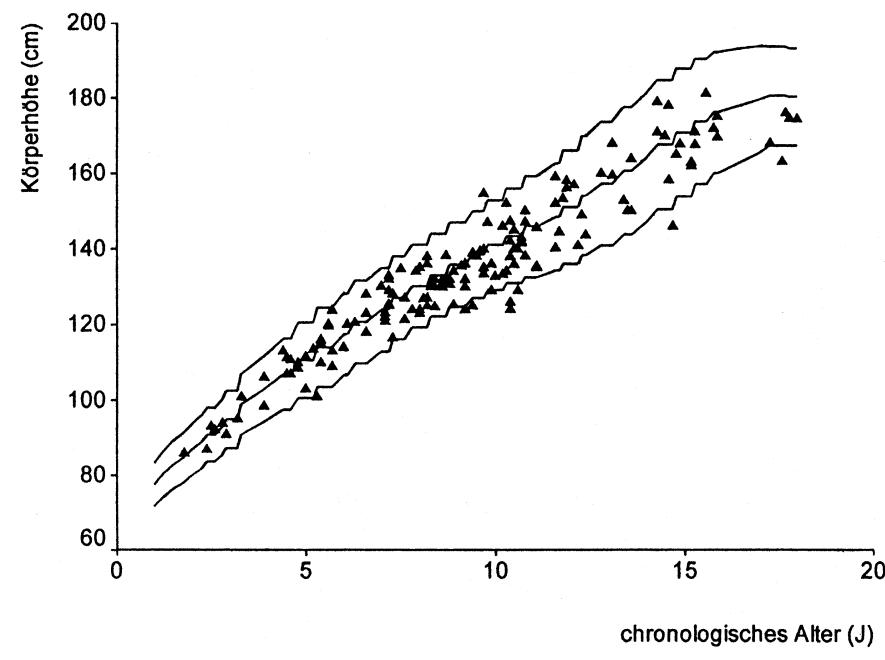

Abb. 1 Körperhöhe asthmakranker Jungen im Vergleich zu den Referenzwerten $(\bar{x} \pm 2 s)$ nach Hesse [31].

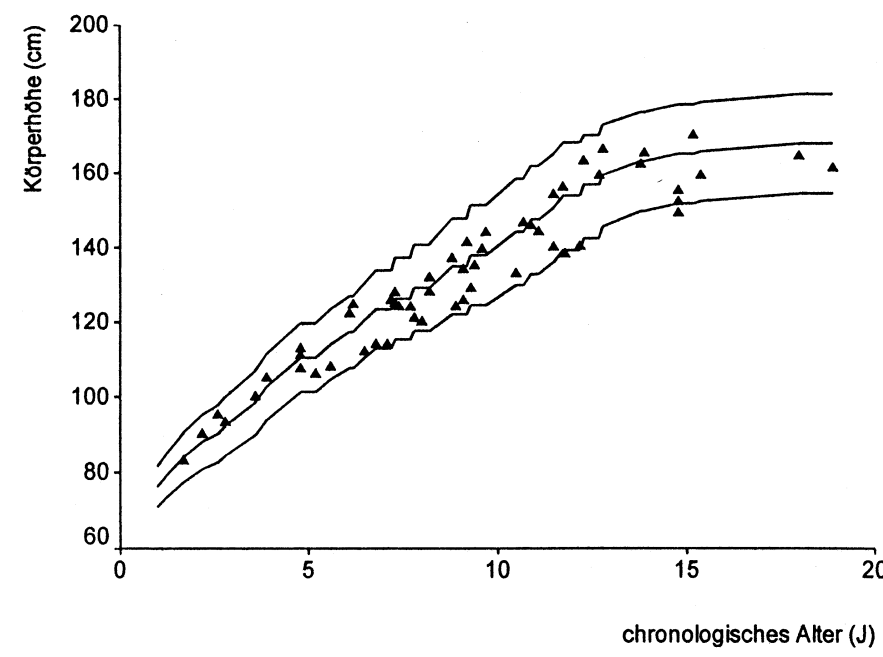

Abb. 2 Körperhöhe asthmakranker Mädchen im Vergleich zu den Referenzwerten $(\bar{x} \pm 2 \mathrm{~s})$ nach Hesse [31].

Die Abb. 3 und $\mathbf{4}$ veranschaulichen die Relation von Knochenund Längenalter zum chronologischen Alter. Nur in den Altersgruppen der Ein- bis Vierjährigen besteht eine gute Übereinstimmung. Dann entwickelt sich eine Retardierung des Knochen- und Längenalters, die über den gesamten Beobachtungszeitraum bestehen bleibt. Von dieser Wachtumsverzögerung ist das KA zunächst etwas stärker betroffen. In den zahlenmäßig kleinen Al-

Tab. 1 Körperhöhe $(\bar{x} \pm s)$ und Körperhöhe-SDS-Werte $(\bar{x} \pm s)$ asthmakranker Kinder in Abhängigkeit vom Erkrankungsschweregrad

\begin{tabular}{|c|c|c|c|c|c|c|}
\hline Schweregrad & $\begin{array}{l}\text { Jungen } \\
n\end{array}$ & $K H(\mathrm{~cm})$ & $K H-S D S$ & $\begin{array}{l}\text { Mädchen } \\
n\end{array}$ & $K H(\mathrm{~cm})$ & $K H-S D S$ \\
\hline 1 & 30 & $140,9 \pm 20,2$ & $-0,41 \pm 1,1$ & 10 & $146,7 \pm 23,6$ & $0,46 \pm 0,5$ \\
\hline 2 & 105 & $133,8 \pm 20,7$ & $-0,17 \pm 1,0$ & 40 & $129,7 \pm 20,9$ & $-0,38 \pm 1,1$ \\
\hline 3 & 18 & $131,3 \pm 23,4$ & $-0,59 \pm 1,1$ & 7 & $124,8 \pm 12,3$ & $-0,97 \pm 0,8$ \\
\hline$p$ & & $>0,05$ & $>0,05$ & & $<0,05$ & $<0,05$ \\
\hline
\end{tabular}




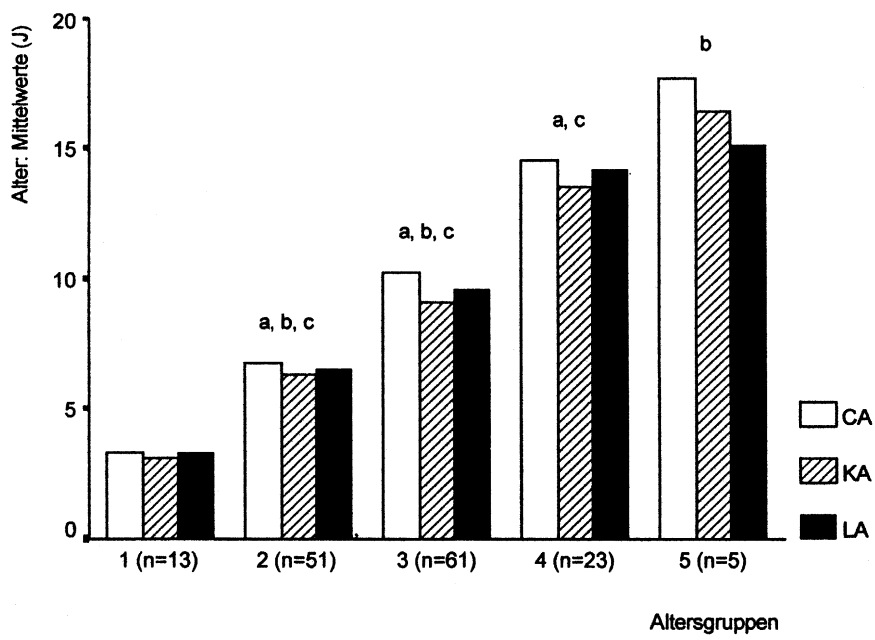

Abb. 3 Chronologisches Alter (CA), Knochen (KA)- und Längenalter (LA) asthmakranker Jungen: Mittelwerte für Vierjahresaltersgruppen a: KA signifikant <CA, b: LA signifikant <CA, c: KA signifikant < LA.

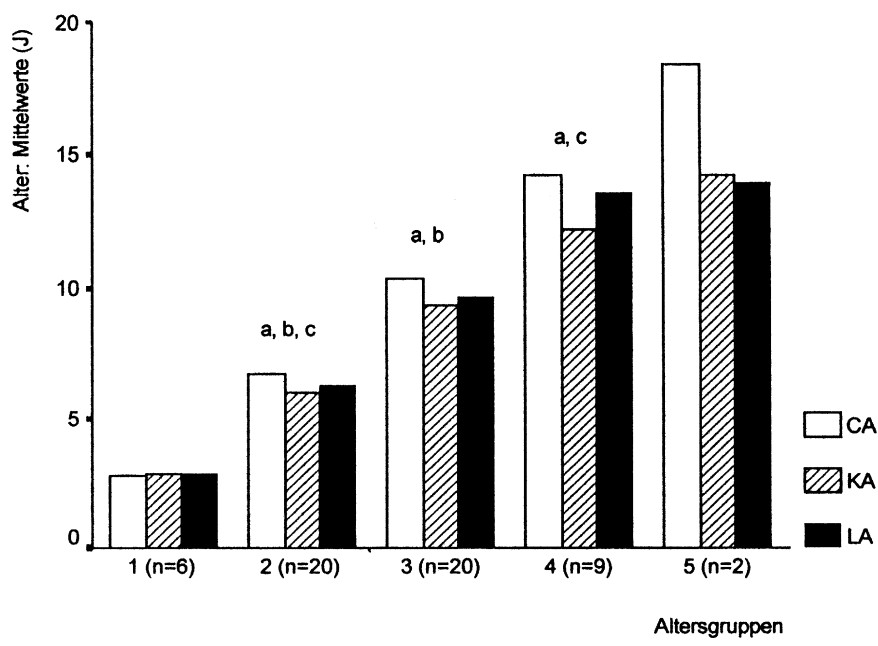

Abb. 4 Chronologisches Alter (CA), Knochen (KA)- und Längenalter (LA) asthmakranker Mädchen: Mittelwerte für Vierjahresaltersgruppen a: KA signifikant <CA, b: LA signifikant < CA, c: KA signifikant < LA.

Tab. 2 Genetische Zielgröße $(\bar{x} \pm s)$ und prognostische Endgröße $(\bar{x} \pm s)$ asthmakranker Kinder in Abhängigkeit vom Erkrankungsschweregrad

\begin{tabular}{|c|c|c|c|c|c|c|c|c|}
\hline \multirow[b]{2}{*}{ Schweregrad } & \multicolumn{4}{|c|}{ Jungen } & \multicolumn{4}{|c|}{ Mädchen } \\
\hline & $n$ & $G Z G(\mathrm{~cm})$ & PEG $(\mathrm{cm})$ & PEG-GZG (cm) & $n$ & $G Z G(\mathrm{~cm})$ & PEG $(\mathrm{cm})$ & PEG-GZG $(\mathrm{cm})$ \\
\hline 1 & 18 & $180,6 \pm 4,2$ & $178,3 \pm 6,8$ & $-2,3 \pm 7,9$ & 3 & $167,4 \pm 1,3$ & $166,1 \pm 9,0$ & $-1,4 \pm 7,7$ \\
\hline 2 & 69 & $180,0 \pm 5,4$ & $179,1 \pm 7,6$ & $-0,9 \pm 5,9$ & 21 & $168,2 \pm 4,5$ & $166,2 \pm 7,0$ & $-1,9 \pm 5,8$ \\
\hline 3 & 12 & $180,8 \pm 6,5$ & $175,8 \pm 6,2$ & $-5,1 \pm 5,2^{*}$ & 6 & $165,9 \pm 5,2$ & $164,9 \pm 5,5$ & $-1,9 \pm 6,1$ \\
\hline gesamt & 99 & $180,2 \pm 5,3$ & $178,6 \pm 7,3$ & $-1,6 \pm 6,3^{* *}$ & 30 & $167,7 \pm 4,4$ & $165,8 \pm 6,8$ & $-1,9 \pm 5,8^{* *}$ \\
\hline
\end{tabular}

KH Referenzwerte für 18-Jährige nach Hesse [31]: Jungen 180,4 $\pm 6,5 \mathrm{~cm}$, Mädchen 167,7 $\pm 6,7 \mathrm{~cm},{ }^{*} \mathrm{p}<0,01,{ }^{*} \mathrm{p}<0,05$

tersgruppen der $\geq 17$-Jährigen dominiert dann aber die Retardierung des Längenalters. Die Unterschiede zwischen CA und KA erwiesen sich für die Altersgruppen 2 bis 4 bei beiden Geschlechtern als statistisch signifikant $(p<0,01)$. Signifikante Differenzen zwischen CA und LA ließen sich in den Altersgruppen $2(p<0,05)$, $3(p<0,01)$ und $5(p<0,05)$ der Jungen sowie $2(p<0,05)$ und 3 $(p<0,01)$ der Mädchen und zwischen LA und KA in den Altersgruppen 2 bis $4(p<0,05)$ der Knaben bzw. $2(p<0,05)$ und 4 $(p<0,01)$ der Mädchen nachweisen.

Ein Vergleich von genetischer Zielgröße und prognostischer Endgröße, der Auskunft über das Vorliegen krankheitsbedingter Wachstumsdefizite geben kann, konnte bei 99 von 153 Jungen, aber nur bei 30 von 57 Mädchen vorgenommen werden. Es zeigte sich, dass die Mittelwerte der PEG bei beiden Geschlechtern in allen Schweregradgruppen ausnahmslos unterhalb der entsprechenden Maßzahlen für die GZG lagen. Die Differenzen zwischen beiden Größen waren für die Schweregradgruppe 3 der Jungen $(180,8 \pm 6,5 \mathrm{zu} 175,8 \pm 6,2 \mathrm{~cm}, \mathrm{p}<0,01)$ und für die Gesamtgruppen (Jungen $180,2 \pm 5,3 \mathrm{~cm}$ zu $178,6 \pm 7,3 \mathrm{~cm}, \mathrm{p}<0,05$, Mädchen $167,7 \pm 4,4 \mathrm{~cm}$ zu $165,8 \pm 6,8 \mathrm{~cm}, \mathrm{p}<0,05)$ signifikant. Es wurde weiterhin deutlich, dass sich die Mittelwerte der PEG ausnahmslos unterhalb des Mittelwertes einer Normalpopulation einordneten, ohne aber die Grenze von $\overline{\mathrm{x}}-\mathrm{s}$ zu unterschreiten (siehe Tab. 2). Im Gegensatz dazu zeigte die mittlere GZG als Ausdruck eines völlig normalen Wachstumspotenzials in allen Schwere- gradgruppen eine sehr gute Übereinstimmung mit den Referenzgruppen für 18-Jährige. Werden beide Größen getrennt betrachtet, lässt sich weder für die PEG noch für die GZG eine signifikante Abhängigkeit vom Erkrankungsschweregrad ausmachen (siehe Tab. 2).

\section{Diskussion}

Von 210 untersuchten asthmakranken Kindern erwiesen sich 9 Jungen (5,9\%) und 2 Mädchen (3,5\%) als kleinwüchsig $(\mathrm{KH}<\overline{\mathrm{X}}-2 \mathrm{~s})$. Da nach dem Studiendesign Kinder kleinwüchsiger Eltern nicht berücksichtigt worden sind, kann als Ursache das Vorliegen eines familiären Kleinwuchses ausgeschlossen werden. Wegen fehlender Daten zur körperlichen Entwicklung der Eltern lassen sich zur Prävalenz einer möglichen konstitutionellen Entwicklungsverzögerung keine Angaben machen. Das gehäufte Auftreten dieser Kleinwuchsform bei kindlichen Asthmatikern ist aber eher als unwahrscheinlich anzusehen. Die erhöhte Kleinwuchsrate ist damit am ehesten in einen kausalen Zusammenhang mit dem Asthma bronchiale bzw. dem dieser Erkrankung oft zugrunde liegenden Atopiestatus zu bringen. Die Ätiologie ist aber noch nicht endgültig geklärt. Diskutiert werden Erkrankungsschweregrad [1 -5], inadäquate Glukokortikoidtherapie [6 - 11,32], chronischer Stress [33] mit Störungen des Nachtschlafes [34] und der nächtlichen Wachstumshormon-Sekretion 
[15,35-39], Hypoxie [40], chronische pulmonale Infektionen $[2,15,33]$, unzureichende bzw. einseitige Ernährung $[2,6,13,14,18,33,41,42]$ und ein erhöhter Grundumsatz [43]. Abgesehen davon, dass alle diese Parameter mehr oder weniger direkt mit dem Erkrankungsschweregrad verbunden sind, ist - mit Ausnahme der Glukokortikoidbehandlung - ein sicherer Beweis für die klinische Relevanz der postulierten Kausalzusammenhänge für das Krankheitsbild des Asthma bronchiale bisher noch nicht erbracht worden. Dagegen darf als gesichert gelten, dass ein schweres Asthma bronchiale - wie jedes andere chronische Leiden auch - Wachstum hemmen kann.

Interessant sind Mitteilungen, nach denen Patienten mit leichtem Krankheitsverlauf und solche, die bislang noch nicht mit Glukokortikoiden behandelt worden sind, ebenfalls erhöhte Kleinwuchsraten aufwiesen $[2,4,6,15]$. Diese Beobachtungen sprechen für eine kausale Bedeutung der Atopie und könnten als Zeichen einer primären atopischen Skelettmanifestation gedeutet werden [17]. Gestützt wird eine solche Annahme durch erhöhte Kleinwuchsraten bei kindlichen Patienten mit anderen Erkrankungen des atopischen Formenkreises, wie atopische Dermatitis [44-46] und allergische Rhinitis [15].

Als markantes klinisches Symptom asthmaassoziierter Wachstums- und Entwicklungsstörungen muss der verzögerte Pubertätseintritt angesehen werden [18,20-22]. Nach den eigenen hier vorliegenden Befunden weisen 1- bis 4-jährige Asthmatiker ein mit dem CA identisches LA und KA auf. Der Retardierungsprozess beginnt bei den 5 - bis 8 -Jährigen. Er lässt sich dann in allen weiteren Altersgruppen nachweisen und ist auch bei den über 17-Jährigen noch nicht rückläufig. Das Ausmaß der Retardierung von KA und LA unterschritt aber zu keinem Zeitpunkt die untere Grenze des engeren Referenzbereiches von $\bar{x} \pm s$. Die übereinstimmende Verzögerung von KA und LA spricht für eine gute Wachstumsprognose. Die in den mittleren Altersgruppen beider Geschlechter im Vergleich zum LA signifikant stärkere Retardierung des KA könnte auf ein methodisches Problem zurückzuführen sein. Zachmann u. Mitarb. konnten zeigen, dass die Bestimmung des Knochenalters nach der von Tanner u. Mitarb. mitgeteilten Methode bei älteren Kindern zu vergleichsweise niedrigen Werten führt [47]. Die Umkehrung der KA-LA-Relation zugunsten des KA in den Altersgruppen der über 17-Jährigen würde als Zeichen eines beginnenden Aufholwachstums gewertet werden können.

Eine ganz ähnliche Entwicklungsdynamik mit verzögert einsetzender Pubertät und normaler Endgröße ist auch von anderen Autoren beschrieben worden. Hauspie u. Mitarb. konnten in einer relativ großen gemischten Longitudinalstudie an 531 asthmakranken Jungen im Alter von 2 bis 20 Jahren zeigen, dass der puberale Wachstumsschub im Mittel 1,3 Jahre verspätet einsetzte und die Schamhaarentwicklung eine Verzögerung von bis zu 2 Jahren aufwies. Nach einer verlängerten Wachstumsphase erreichten die Patienten eine normale Endgröße [18]. Balfour-Lynn, der das Wachstum von 66 chronisch kranken Asthmakindern über einen mittleren Zeitraum von 13,1 Jahren verfolgte, konnte bis zu einem Alter von 10 Jahren keinerlei Abweichungen beobachten, dann jedoch kam es bei 30 Kindern zu einer Verzögerung der Wachstumsgeschwindigkeit, verbunden mit einem verspäteten Pubertätseintritt. Alle erreichten eine normale Endgröße [20]. Martin u. Mitarb., die das Wachstum von 342 Asthma- kindern zwischen dem 7. und 21. Lebensjahr beobacheten, registrierten eine im Alter von 10 Jahren einsetzende Retardierung, die bei 14-Jährigen ihr größtes Ausmaß erreichte, mit 21 Jahren aber nicht mehr nachweisbar war [8]. Norjavaara u. Mitarb. werteten das medizinische Datenmaterial mehrerer schwedischer Musterungsjahrgänge aus und konnten zeigen, dass 18-jährige Asthmatiker eine normale, im Vergleich zu Gesunden aber signifikant niedrigere Körperhöhe erreichen [48]. Shohat u. Mitarb. wiederum stellten nach Analyse der Musterungsbefunde 17-jähriger israelischer Wehrpflichtiger beiderlei Geschlechts fest, dass Asthmatiker mit leichtem Krankheitsverlauf signifikant größer sind als gesunde Kontrollpersonen [24].

Vergleichende Untersuchungen von Erwachsenenendgröße und genetischer Zielgröße sind bislang nur vereinzelt zur Bewertung wachstumsrelevanter Effekte einer Langzeittherapie mit inhalativen Glukokortikoiden vorgenommen worden. Es konnte gezeigt werden, dass Asthmatiker unabhängig von einer Behandlung mit diesen Medikamenten eine normale und mit der genetischen Zielgröße übereinstimmende Endgröße erreichen [25-27]. Durch die von uns vorgenommene Berechnung der PEG lässt sich die gute Prognose asthmaassoziierter Wachstumsstörungen bestätigen. Unabhängig vom Erkrankungsschweregrad lagen die mittleren PEG-Werte alle im engeren Referenzbereich von $\bar{x}-1 \mathrm{~s}$, allerdings ausnahmslos unterhalb des Mittelwertes der Normalpopulation (siehe Tab. 2) und - im Unterschied zum Schrifttum - auch unterhalb der GZG. Für die Gesamtgruppen beider Geschlechter und die Schweregradgruppe 3 der Jungen erwiesen sich die Differenzen als statistisch signifikant.

Kindliche Asthmatiker besitzen somit ein normales Wachstumspotenzial, das aber von einem Teil der Patienten nicht voll ausgeschöpft werden kann. Dafür scheint - wie die relativ niedrigen PEG-Mittelwerte in den Schweregradgruppen 3 beider Geschlechter zeigen - teilweise der Erkrankungsschweregrad verantwortlich zu sein. Nach dem gewählten Studiendesign (Definition des Schweregrades über die medikamentöse Therapie) muss auch ein Glukokortikoid-Effekt in Betracht gezogen werden. Das Vorliegen anderer Kausalzusammenhänge kann jedoch nicht ausgeschlossen werden. So könnte das Wachstumsdefizit auch auf einer atopiebedingten primären Störung der Skelettreifung beruhen [17]. Dafür spricht die Tatsache, dass die Mittelwerte der PEG in den einzelnen Schweregradgruppen konstant unter den GZGWerten lagen und keine signifikanten Unterschiede aufwiesen.

Wird die Differenz von GZG und PEG betrachtet, durch die der Einfluss der Asthmaerkrankung bzw. der Atopie auf das Wachstum abgeschätzt werden kann, dann unterscheiden sich die eigenen Ergebnisse deutlich von den Angaben des Schrifttums. Im Unterschied zu den Mitteilungen von Agertoft u. Mitarb., Silverstein u. Mitarb. und van Bever u. Mitarb. [25-27] konnten wir bei beiden Geschlechtern eine im Vergleich zur GZG signifikant erniedrigte PEG nachweisen. Die Abweichung basiert nicht auf Differenzen in der Berechnung der Endgröße, sondern auf der Verwendung unterschiedlicher Formeln zur Ermittlung der GZG. Um der Akzeleration Rechnung zu tragen, erfolgte in der vorliegenden Arbeit die Bestimmung der GZG bei Knaben durch Addition von $10,2 \mathrm{~cm}$ zur und bei Mädchen durch Subtraktion von $2,6 \mathrm{~cm}$ von der mittleren Elterngröße [30]. Die sehr gute Übereinstimmung der so berechneten GZG mit den Referenzdaten von Hesse [31] spricht für Aktualität und Korrektheit dieser 
Berechnungsgrundlage. Sowohl Agertoft u. Mitarb. als auch van Bever u. Mitarb. und Silverstein u. Mitarb. verwendeten andere Korrekturfaktoren und ermittelten so deutlich niedrigere genetische Zielgrößen [25-27]. Daraus resultiert eine relative Zunahme der auf die GZG bezogenen Endgröße. Es ist deshalb fraglich, ob asthmakranke Kinder als Gruppe tatsächlich eine über der GZG liegende Endgröße erreichen. Unsere Ergebnisse sprechen dafür, dass kindliche Asthmatiker in der Regel eine normale Endgröße erlangen, die aber eher unterhalb des Mittelwertes einer Referenzpopulation im Bereich $\bar{x}-1 \mathrm{~s}$ und unterhalb der GZG liegt. Das deutet auf eine Wachstumsbeeinflussung durch das Asthma bronchiale bzw. die dieser Erkrankung oft zugrunde liegende Atopie hin.

\section{Literatur}

${ }^{1}$ Faliers CJ, Tan LS, Szentivanyi J, Jorgensen JR, Bukantz SC. Childhood asthma and steroid therapy as influences on growth. Am J Dis Child 1963; 105: $127-137$

2 Murray AB, Fraser BM, Hardwick DF, Pirie GE. Chronic asthma and growth failure in children. Lancet 1976; 7978: 197-198

3 Smith JM. Prolonged treatment with prednisolone in children with asthma. Tubercle 1963; 44: $281-284$

${ }^{4}$ Snyder RD, Collipp PJ, Greene JS. Growth and ultimate height of children with asthma. Clin Pediatr 1967; 6: 389-392

${ }^{5}$ Wittig HJ, McLaughlin ED, Belloit JD. Growth retardation in children with chronic asthma in the absence of prolonged steroid therapy. Allergol Immunpathol 1978; 6: 203-208

${ }^{6}$ Falliers CJ, Szentivanyi J, McBride M, Bukantz SC. Growth rate of children with intractable asthma: observations on the influence of the illness and its therapy with steroids. J Allergy 1961; 32: 420-434

${ }^{7}$ Kerrebijn KF, DeKroon JPM. Effects on height of corticosteroid therapy in asthmatic children. Arch Dis Child 1968; 43: 556- 561

${ }^{8}$ Martin AJ, Landau LI, Phelan PD. The effect on growth of childhood asthma. A Pediatr Scand 1981; 70: 683-688

${ }^{9}$ van Metre TE, Niermann WA, Rosen LJ. A comparison of the growth suppressive effect of cortisone, prednisone, and other adrenal cortical hormones. J Allergy Clin Immunol 1960; 31: 531 - 542

${ }^{10}$ Morris HG. Growth and skeletal maturation in asthmatic children: effect of corticosteroid treatment. Pediatr Res 1975; 9: 579-583

11 Oberger E, Engstrom I, Karlberg J. Long-term treatment with glucocorticoids/ACTH in asthmatic children. III Effects on growth and adult height. A Pediatr Scand 1990; 79: 77-83

12 Spock A. Growth patterns in 200 children with bronchial asthma. Ann Allergy 1965; 23: $608-615$

${ }^{13}$ Klein G, Duriseti L, Miyamoto S, Writht D, Galant SP. Nutrition and growth in the nonsteroid-dependent asthmatic child. J Allergy Clin Immunol 1978; 61: 158-159

${ }^{14}$ Klein GL, Dungy CI, Galant SP. Growth and the nutritional status of nonsteroid-dependent asthmatic children. Ann Allergy 1991; 67: $80-84$

${ }^{15}$ Ferguson AC, Murray AB, Tze WJ. Short stature and delayed skeletal maturation in children with allergic disease. J Allergy Clin Immunol 1982; 69: 461 - 466

${ }^{16}$ Cohen MB, Weller RR, Cohen S. Anthropometry in children: progress in allergic children as shown by incriments in height, weight and maturity. Am J Dis Child 1940; 60: 1058 - 1066

${ }^{17}$ Baum WF, Klöditz E, Thiemann HH. Die atopische Skelettretardierung als eine mögliche Ursache für Kleinwuchs und Brustkorbdeformierung asthmakranker Kinder. Kinderärztl Praxis 1993; 61: 285-290

${ }^{18}$ Hauspie R, Susanne C, Alexander F. Maturational delay and temporal growth retardation in asthmatic boys. J Allergy Clin Immunol 1977; 59: $200-206$

${ }^{19}$ Maiello N, Del Guidice MM, Marano C, Capristo A, Salzano V. Rilievi sulla maturazione scheletrica e sull'accrescimento staturale in bambini asmatici in rapporto alla terapia cortisonica. Pediatria 1980; 88: $61-80$

${ }^{20}$ Balfour-Lynn L. Growth and childhood asthma. Arch Dis Child 1986; 61: 1049-1055

${ }^{21}$ Balfour-Lynn L. Effect of asthma on growth and puberty. Pediatrician 1987; 14: $237-241$
${ }^{22}$ Hauspie R, Susanne C, Alexander F. A mixed longitudinal study of the growth in height and weight in asthmatic children. Hum Biol 1976; 48: $271-283$

${ }^{23}$ Inoue T, Doi S, Takamatsu I, Murayama N, Kameda M, Toyoshima K. Effect of long-term treatment with inhaled Beclomethasone diproprionate on growth of asthmatic children. J Asthma 1999; 36: 159-164

${ }^{24}$ Shohat M, Shohat T, Kedem R, Mimouni M, Danon YL. Childhood asthma and growth outcome. Arch Dis Child 1987; 62: 63-65

${ }^{25}$ Agertoff L, Pedersen S. Effect of long-term treatment with inhaled Budenoside on adult height in children with asthma. N Engl J Med 2000; 343: $1064-1069$

${ }^{26}$ VanBever HP, Desager KN, Lijssens N, Weyler JJ, Du Caju MVL. Does treatment of asthmatic children with inhaled corticosteroids affect their adult height? Ped Pneumol 1999; 27: 369-375

27 Silverstein MD, Yunginger JW, Reed CE, Petterson T, Zimmermann D, Li JT, Fallon WM. Attained adult height after childhood asthma; effect of glucocorticoid therapy. J Allergy Clin Immunol 1997; 99: 466-474

${ }^{28}$ Martin R, Saller K. Lehrbuch der Anthropologie. Stuttgart: Fischer, 1957: $323-324$

${ }^{29}$ Tanner JM, Whitehouse RH, Marshall WA, Healy MJR, Goldstein H. Assessment of skeletal maturity and prediction of adult height. London: Academic Press, 1975

${ }^{30}$ Sippell WG. Störungen des Wachstums und der Entwicklung. In: Betke K, Külzer W, Schaub J (Hrsg). Lehrbuch der Kinderheilkunde. Stuttgart: Thieme, 1991: 219-234

${ }^{31}$ Hesse V. Wachstum und Reifung. In: Meng W, Ziegler R (Hrsg). Endokrinologie Grundlagen, Klinik, Praxis. Jena, Stuttgart: Fischer, 1997: $105-131$

${ }^{32}$ Allen DB, Mullen M, Mullen B. A meta-analysis of the effect of oral and inhaled corticosteroids on growth. J Allergy Clin Immunol 1994; 83: $196-199$

33 Preece MA, Law CM, Davies PSW. The growth of children with chronic paediatric disease. Clin Endocrinol 1986; 15: 453-477

${ }^{34}$ Kales A, Kales JD, Sly RM, Scherf MB, Tan TL, Preston TA. Sleep patterns of asthmatic children: allnight electroencephalographic studies. J Allergy Clin Immunol 1970; 46: 300-308

35 Crowley S, Hindmarsh PC, Matthews DR, Brook CGD. Growth and the growth hormone axis in prepubertal children with asthma. J Pediatr 1995; 126: $297-303$

${ }^{36}$ Eastman CJ, Lazarus L. Growth hormone release during sleep in growth retarded children. Arch Dis Child 1973; 48: 502-507

37 Baum WF, Klöditz E, Hesse V, Jahreis G, Schneyer U, Giebler H. Steigerung der spontanen Wachstumshormon-Sekretion bei asthmakranken Kindern - ein Symptom der atopischen Disposition? Kinderärztl Praxis 1993; 61: $323-328$

38 Nigam P, Laungia S, Goyal G, Dutt B. Growth hormone secretion in growth retarded asthmatic children. Indian J Pediatr 1977; 44: $315-319$

${ }^{39}$ Sanders SS, Norman AP. Growth hormone secretion in growth-retarded asthmatic children. Br Med J 1969; 3: 25-26

${ }^{40}$ Smith TF, Hudgel DW. Arterial oxygen desaturation during sleep in children with asthma and its relation to airway obstruction and ventilatory drive. Pediatrics 1980; 66: 746-751

${ }^{41}$ Cogswell JJ, El-Bishti MM. Growth retardation in asthma: role of caloric deficiency. Arch Dis Child 1982; 57: 473-475

${ }^{42}$ Goldey DH, Mansmann HC, Rasmussen Al. Zinc status of asthmatic, prednisone-treated asthmatic, and non-asthmatic children. J Am Diet Assoc 1984; 84: 157-163

${ }^{43}$ Zeitlin SR, Bond S, Wootton S, Gregson RK, Radford M. Increased resting energy expenditure in childhood asthma: does this contribute toward growth failure? Arch Dis Child 1992; 67: 1366 - 1369

${ }^{44}$ Kristmundsdottir F, David TJ. Growth impairment in children with atopic eczema. J R Soc Med 1987; 80: 9-12

45 Pike MG, Chang CL, Atherton DJ, Carpenter RG, Preece MA. Growth in atopic eczema: a controlled study by questionaire. Arch Dis Child 1989; 64: $1566-1569$

${ }^{46}$ Massarano AA, Hollis S, Devlin J, David TJ. Growth in atopic eczema. Arch Dis Child 1993; 68: 677-679

${ }^{47}$ Zachmann M, Sobradillo B, Frank M, Frisch H, Prader A. Bayley-Pinneau, Roche-Wainer-Thissen and Tanner height predictions in normal children and in patients with various pathologic conditions. J Pediatr 1978; 93: 749-755

${ }^{48}$ Norjavaara E, Gerhardsson M, Lindmark B. Reduced height in swedish men with asthma at the age of conscription for military service. J Pediatr 2000; 137: 25-29 